\title{
ESTUDIOS ESPORALES EN ESPECIES DEL GRUPO BLECHNUM PENNA-MARINA (BLECHNACEAE-PTERIDOPHYTA)
}

\author{
Lilian M. PASSARELLI \\ Laboratorio de Estudios de Anatomía Vegetal Evolutiva y Sistemática (LEAVES), \\ Facultad de Ciencias Naturales y Museo de La Plata, 64 entre 120 y diagonal 113, \\ B1904 DZB.Argentina \\ lmpassarelli@yahoo.com.ar
}

Recibido el 29 de junio de 2007, aceptado para publicación el 26 de septiembre de 2007

Publicado "on line" en octubre de 2007

\begin{abstract}
Study of the spores of species of the Blechnum penna-marina group (BlechnaceaePteridophyta). The spores of eleven taxa of the Blechnum penna-marina group, B.asperum, $B$. blechnoides, B. corralense, B. fernandezianum, B. microphyllum, B. mochaenum subsp. achalense, $B$. mochaenum subsp. mochaenum, B. mochaenum subsp. squamipes, B. penna-marina, $B$. spicant, and $B$. stoloniferum were studied with light, and scanning electron microscopes. Spores are monolete and ellipsoidal in all taxa, with macro-ornamentation predominantly among muriform types. Sporoderm consists of a psilate, rugulate, or more or less supperficially granulate exospore, and a rugate, rugulate, venulose-rugulate, fossulate-rugulate, cristate-reticulate perispore. An exception are B. fernandezianum spores, with psilate perispores that bear orbicules. The perispore is a stratified, rather complex wall formed by three strata of different thickness and morphology: an outer continuous stratum, which bears the elements of the sculpture, a middle stratum which is alveolate, foliose or bear pillar-like elements, and a very thin, not clearly defined inner stratum. Blechnum mochaenum subsp. mochaenum and B. penna-marina showed larger spores in some specimens, a condition that may be related with different ploidia. Macro-ornamentation and morphology of the perispore of some taxa is also found in species of other groups of Blechnum. Performed studies suggest that spore characters do not define a particular group but the taxa, which is considered a new promising perspective for the systematic of the whole genus.
\end{abstract}

Key words. Blechnaceae, Blechnum, B. penna-marina group, spores, morphology, systematics

RESUMEN. Estudios esporales en especies del grupo Blechnum penna-marina (BlechnaceaePteridophyta). Las esporas de once taxones del grupo B. penna marina, B. asperum, B. blechnoides, $B$. corralense, $B$. fernandezianum, B. microphyllum, B. mochaenum subsp. achalense, $B$. mochaenum subsp. mochaenum, B. mochaenum subsp. squamipes, $B$. penna-marina, $B$. spicant y $B$. stoloniferum se estudiaron con microscopio de luz y electrónico de barrido. Las esporas son monoletes y elipsoidales en todos los taxones, con macro-ornamentación predominantemente muriforme. El esporodermo consiste de un exosporio psilado, rugulado o con superficie granular y un perisporio rugado, rugulado, venuloso-rugulado, fosulado-rugulado o crestado-reticulado. Una excepción son las esporas de $B$. fernandezianum, con perisporios psilados con orbículas. El perisporio es estratificado, con capas de diferente morfología y espesor: una capa externa continua, que soporta los elementos de la escultura, una media, alveolar, foliosa o con elementos columnares y una interna muy delgada que no siempre se distingue claramente. Algunos ejemplares de B. mochaenum subsp. mochaenum y B. penna-marina 
mostraron esporas más grandes en algunos ejemplares, lo que podría relacionarse con diferentes niveles de ploidía. La macro-ornamentación de las esporas y la morfología de las capas observadas en algunos taxones se encuentra también en esporas de otros grupos del género. Los estudios llevados a cabo sugieren que los rasgos esporales no definen un grupo particular sino taxones, una conclusión que abre perspectivas prometedoras para la sistemática del género.

Palabras clave. Blechnaceae, Blechnum, grupo B. penna-marina, esporas, morfologia, sistemática.

\section{INTRODUCCIÓN}

El género Blechnum tiene una distribución amplia y crece en América, África, Magadascar, Europa, Melanesia, Micronesia, hacia el E hasta Japón, Australia, Nueva Zelanda, Polinesia hasta Tahití, Rapa e islas Marquesas y es adventicio en Hawái. Incluye unas 150 especies, de las cuales unos 92 taxones habitan en Sudamérica y Mesoamérica (Rolleri \& Prada, 2006 a), en selvas húmedas, bajas o montanas, bordes de selvas o bosques, matorrales y pajonales de cierta altura sobre el nivel del mar, generalmente inundables y también sabanas o áreas perturbadas. En Argentina crecen unas 20-21 especies (Durán, 1997; Ponce, 1996) que se distribuyen en el NO, NE, las Sierras Australes de Buenos Aires, las Sierras Centrales, los bosques hiperhúmedos andinopatagónicos, Tierra del Fuego y las islas Malvinas.

Tryon \& Tryon (1982) lo subdividieron en siete grupos informales, cada uno con el nombre de una especie representativa: grupo B. buchtienii Rosenst., grupo B. fragile (Liebm.) C. V. Morton \& Lellinger, grupo B. lineatum (Sw.) C. Chr., grupo B. loxense (Kunth) Hook. ex Salomon, grupo $B$. occidentale L., grupo B. penna-marina (Poir.) Kuhn y grupo B. serrulatum Rich., sobre la base de los caracteres de sus ejes y frondas. No consideraron los caracteres de las esporas, aunque, posteriormente Tryon \& Lugardon (1991) ilustraron, con microscopía electrónica de barrido treinta especies procedentes de toda el área de distribución del género e incluyeron en ese trabajo las de B. penna-marina.

En relación con el grupo $B$. pennamarina hay pocos estudios esporales adicionales. Lugardon $(1965,1974)$ analizó las esporas de $B$. spicant L. mediante microscopios de luz y electrónico de transmisión y de barrido, desde la meiosis a la madurez; Morbelli (1976) ilustró, mediante microscopio de luz, las esporas de B. microphyllum (Goldm.) C.V. Morton, $B$. mochaenum G. Kunkel y B. penna-marina, Durán (1997) describió las esporas de las especies argentinas de 'Blechnum y describió los perisporios lisos a "subpunteados" para B. penna-marina, lisos para $B$. microphyllum y "punteados" para B. mochaenum, pero no suministró medidas ni ilustraciones. Ramos Giacosa et al. (2006 a-b) estudiaron las esporas de algunas especies de Blechnum de la Argentina, describiendo su morfología en forma general y sin referirse particularmente a su implicancia en la sistemática de esas especies.

Rolleri \& Prada (2006 b) revisaron el grupo B. penna-marina y lo caracterizaron por reunir plantas con esporófitos más bien pequeños, perennes, coloniales, con rizomas escamosos y estoloníferos, estípites papilosos de sección cóncavo-convexa, con un solo surco adaxial y tres haces vasculares, láminas pinnatífidas o pinnatisectas con segmentos adnatos al raquis, indumento superficial representado por pelos uniseriados pluricelulares, grandes hidatodos epifílicos activos, esporas con perisporios lisos a rugulados y reproducción vegetativa 
por medio de estolones. Esas autoras analizaron por primera vez la morfología esporal en relación con otros rasgos de afinidad de las especies y consideraron que la morfología esporal podría aportar caracteres importantes en la sistemática del género.

En este trabajo se analizan las esporas de once taxones del grupo B. penna-marina, tal como fue definido por Rolleri \& Prada (2006 b), con el objetivo de ampliar el conocimiento palinológico del género e investigar la relación eventual entre la morfología de las esporas y los grupos de especies afines.

Los taxones estudiados son B. asperum (Klotzsch) J.W. Sturm., B. blechnoides (Bory) Keyserl., B. corralense Espinosa, $B$. fernandezianum (Looser) Prada \& Rolleri, B. microphyllum, B. mochaenum subsp. achalense (Hieron.) Prada \& Rolleri , $B$. mochaenum G. Kunkel subsp. mochaenum, B. mochaenum subsp. squamipes (Hieron.) Prada \& Rolleri, B. penna-marina, B. spicant y B. stoloniferum (Mett. ex E. Fourn) C. Chr. Las esporas se describen detalladamente y se analiza y discute el valor diagnóstico de los rasgos esporales en el grupo y en el género.

\section{MATERIALES Y MÉTODOS}

Los estudios se efectuaron en material de herbario procedente de los herbarios CONC, LIL, LP, MA, MACB y US (Holmgren et al., 1990), mediante microscopía de luz (ML) y electrónica de barrido (MEB). Blechnum asperum se estudió solamente con ML, debido a la escasez de material fértil disponible para el análisis. Se investigaron todos los ejemplares fértiles incluidos en el trabajo de Rolleri \& Prada (2006 b); aquí se suministra una selección de ejemplares de referencia, a continuación de la descripción de cada taxón.

Las muestras de esporas se tomaron de especímenes de todas las localidades de cada taxón y se seleccionaron de plantas de diferentes tamaños. En todos los ejemplares se midieron los diámetros ecuatoriales mayor y menor y los valores resultantes se expresan en $\mu \mathrm{m}$. Las esporas se tomaron de las pinnas fértiles mediante una aguja histológica o un pincel fino, se montaron sin ningún tratamiento sobre bases de vidrio de $3 \times 3$ $\mathrm{mm}$, con cinta adhesiva de doble faz y se metalizaron con oro en un metalizador JEOL JFC-1100. Las observaciones se efectuaron con un microscopio electrónico Jeol /EO JSM 6360, en el Servicio de Microscopía Electrónica de la Facultad de Ciencias Naturales y Museo de La Plata.

La terminología relacionada con las esporas se tomó de Lellinger \& Taylor (1997) y Lellinger (2002), aunque en algunos casos se efectuaron modificaciones o combinaciones de los términos de esos autores.

\section{RESULTADOS}

Las esporas de los taxones del grupo $B$. penna marina son elipsoidales, con una relación diámetro mayor / diámetro menor entre 1,1 y 2 (Tabla 1). Miden 53 x $41 \mu \mathrm{m}$ en B. asperum, 49 x $40 \mu \mathrm{m}$ en B. blechnoides; $57 \times 36 \mu \mathrm{m}$ en $B$. corralense; $50 \times 36 \mu \mathrm{m}$ en B. fernandezianum; 48 × $30 \mu \mathrm{m}$ en $B$. microphyllum; 54 × $35 \mu \mathrm{m}$ y 44 x $30 \mu \mathrm{m}$ en B. mochaenum subsp. mochaenum; $50 \times 33$ $\mu \mathrm{m}$ en B. mochaenum subsp. achalense; 45 x $32 \mu \mathrm{m}$ en B. mochaenum subsp. squamipes; 48 × $36 \mu \mathrm{m}$ y 44 × 31, en $B$. penna marina; $50 \times 35 \mu \mathrm{m}$ en $B$. spicant y 51 × $36 \mu \mathrm{m}$ en $B$. stoloniferum. Son monoletes, la lesura tiene desde 20 a $60 \mu \mathrm{m}$ y es tenuimarginada, suavemente curva o sobresaliente.

El esporodermo es castaño-amarillento y está formado por exosporio y perisporio. 


\begin{tabular}{|c|c|c|c|c|c|c|c|}
\hline 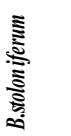 & 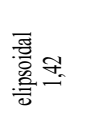 & 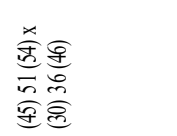 & 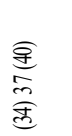 & 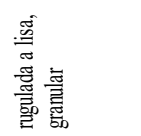 & 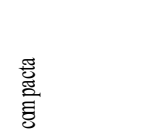 & 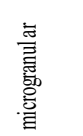 & 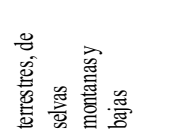 \\
\hline 彦 & $\begin{array}{l}\text { 品 } \\
\text { 总蛋 }\end{array}$ & 虏 & 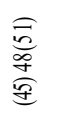 & 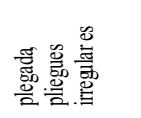 & 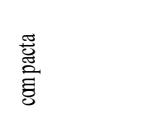 & $\stackrel{̊}{=}$ & 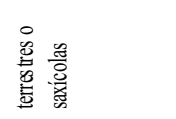 \\
\hline 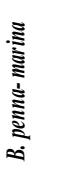 & 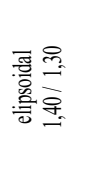 & 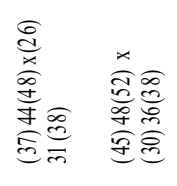 & $\begin{array}{l}\frac{f}{c} \\
\stackrel{2}{a} \\
\stackrel{2}{a}\end{array}$ & 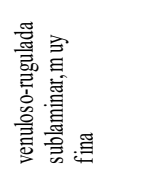 & 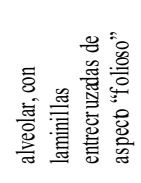 & 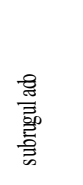 & 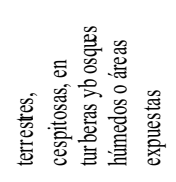 \\
\hline 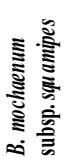 & 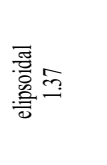 & 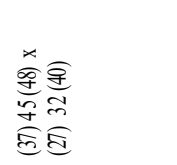 & 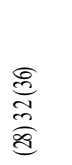 & 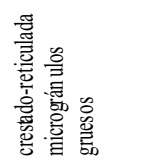 & 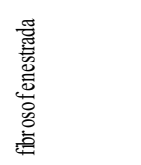 & 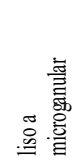 & 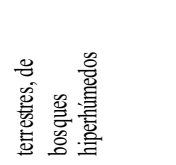 \\
\hline 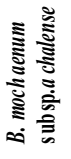 & 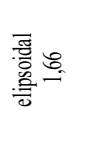 & 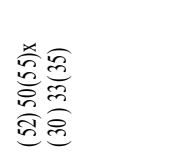 & $\frac{\sqrt{2}}{\frac{a}{a}}$ & 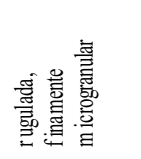 & 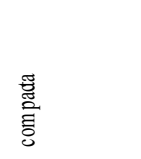 & $\stackrel{\circ}{=}$ & 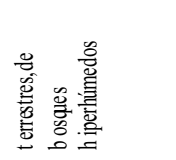 \\
\hline 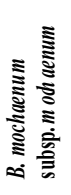 & 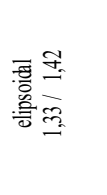 & 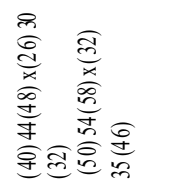 & 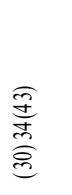 & 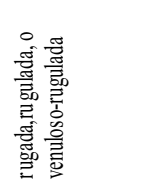 & 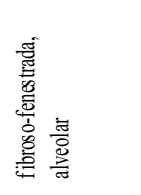 & $\stackrel{\circ}{=}$ & 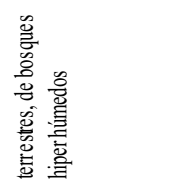 \\
\hline 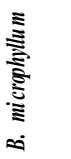 & 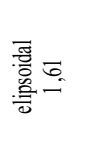 & 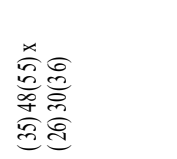 & 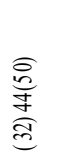 & 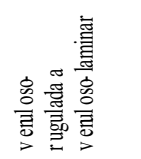 & 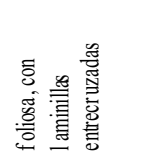 & 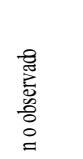 & 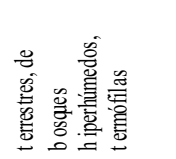 \\
\hline 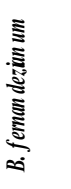 & 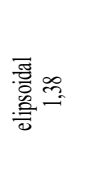 & 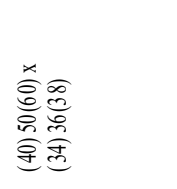 & 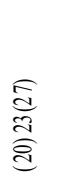 & 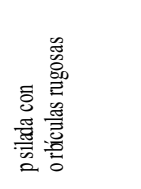 & 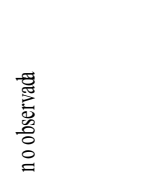 & $\stackrel{\circ}{=}$ & 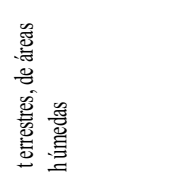 \\
\hline 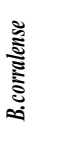 & 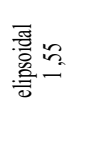 & 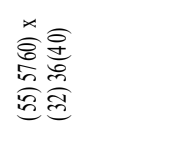 & 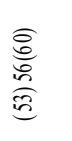 & 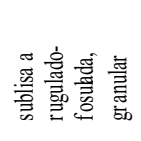 & 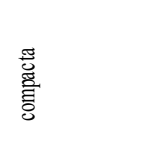 & 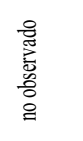 & 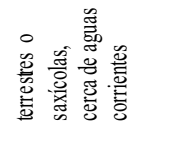 \\
\hline 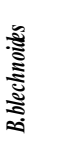 & 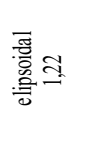 & 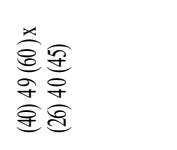 & 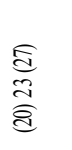 & 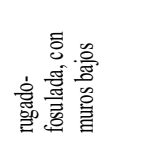 & 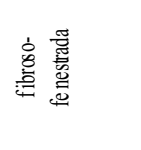 & 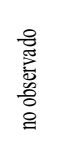 & 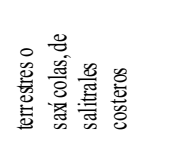 \\
\hline 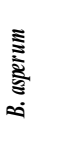 & 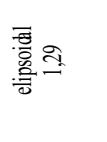 & 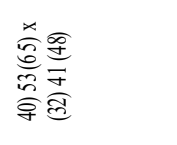 & $\underset{\sqrt{a}}{\stackrel{0}{a}}$ & 点 & 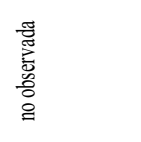 & $\stackrel{\circ}{=}$ & 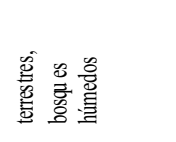 \\
\hline 产 & 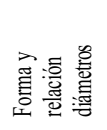 & 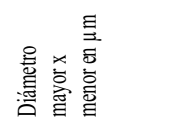 & 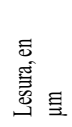 & 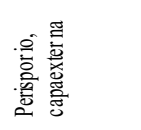 & 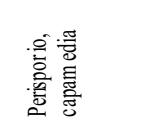 & 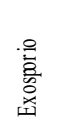 & 莺 \\
\hline
\end{tabular}




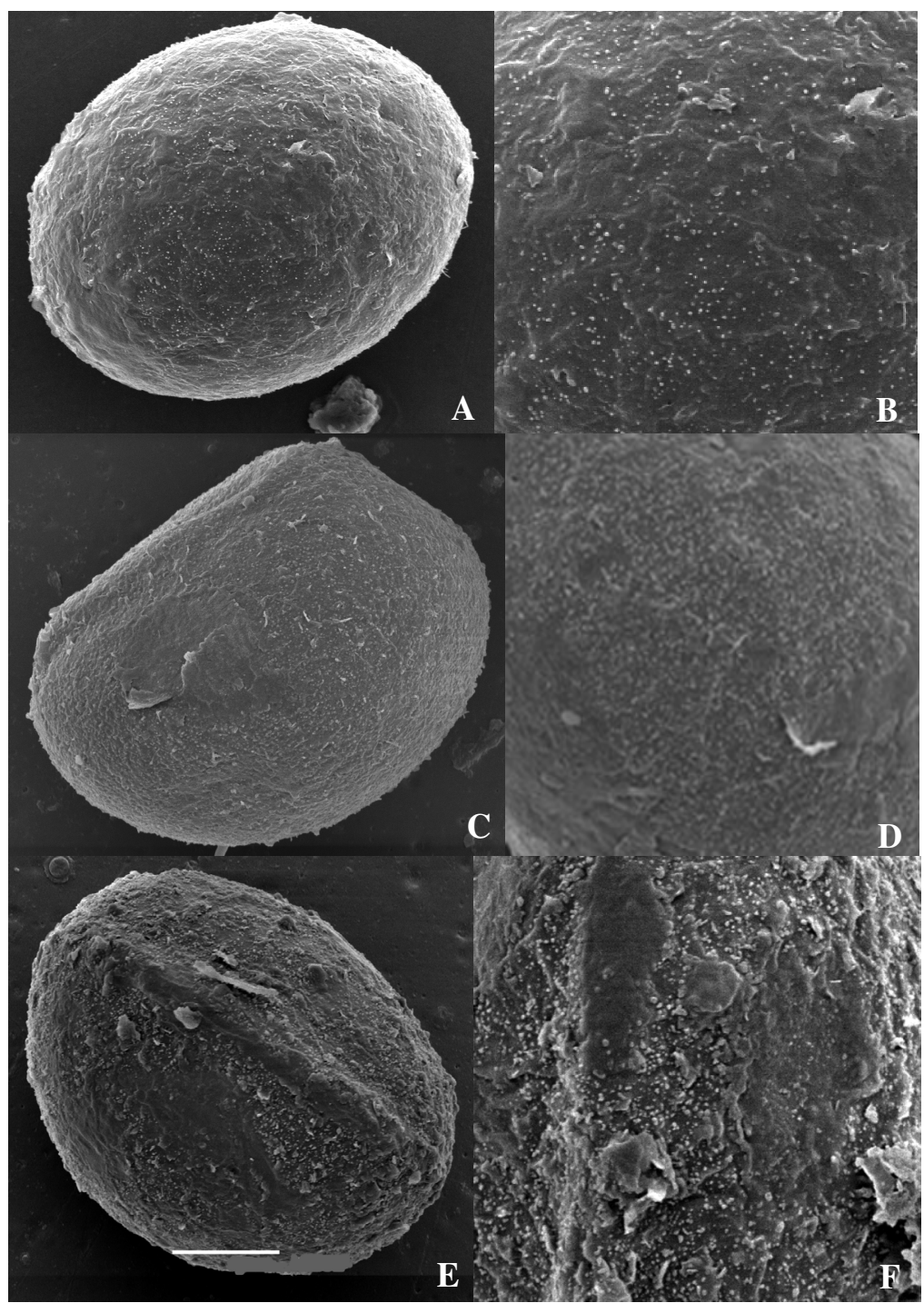

Figura 1. Caracteres esporales en el grupo Blechnum penna-marina: B. corralense, B. mochaenum subsp. achalense y B. stoloniferum. A-B: B. corralense, de Chile, Valdivia, Gunckel 1961(LIL). A: Vista polar, con perisporio. B: Vista polar distal, con perisporio. C-D: B. mochaenum subsp. achalense, de Neuquén, Núñez s.n. (BA 56522). C: Vista lateral, con perisporio, lesura labiada. D: Vista ecuatorial. E-F: B. stoloniferum, de México, Oaxaca, Mickel \& Leonard 4661 (LP). E: Vista polar proximal. Perisporio liso con microgránulos. F: Detalle de perisporio con microgránulos. Barra: $10 \mu \mathrm{m}$ en A, C, $\mathrm{E} ; 5 \mu \mathrm{m}$ en B, D, F. Characters of the spores of the Blechnum penna-marina group: B. corralense, $B$. mochaenum subsp. achalense and B. stoloniferum. A-B B. corralense, from Chile, Valdivia, Gunckel 1961(LIL). A: Spore with perispore, polar view. B: Spore with perispore, polar view, $C-D: B$. mochaenum subsp. achalense, from Neuquén, Núnez s. n. (BA 56522). C: Spore with perispore, lateral view and labiate laesura. D: The same, an equatorial view. E-F: B. stoloniferum, from México, Oaxaca, Mickel \& Leonard 4661 (LP). E: Polar proximal view. Smooth perispore with micro-granules. F: Detail of the perispore with micro-granules. Bar: 10 um en $A, C, E ; 5$ m en $B, D, F$. 
E1 exosporio presenta escasa variación; generalmente es psilado en la mayoría de los taxones aunque en algunos se observaron superficies microgranuladas (B. penna marina y B. stoloniferum).

El perisporio tiene ornamentación variable y hay perisporios sublisos a rugulados o fosulado- rugulados (B. corralense, $B$. mochaenum subsp. achalense, $B$. stoloniferum), venuloso-rugulados ( $B$. mochaenum subsp. mochaenum), rugados, con cierta variación en la la profundidad de las microarrugas (B. blechnoides), rugulados y venuloso-laminares, quebradizos, que se desprenden con facilidad en laminillas finas y dejan ver una capa más complejamente ornamentada por debajo (B. microphyllum y $B$. penna-marina), plegados, con pliegues gruesos o delgados, regulares o irregulares ( $B$. spicant), rugulado-crestados, con crestas bajas e irregulares (B. asperum) y crestadoreticulados (B. mochaenum subsp. squamipes). Blechnum fernandezianum, finalmente, se destaca por los perisporios psilados con orbículas dispersas en grupos irregulares.

El perisporio es estratificado, con dos ó tres capas de diferente espesor y morfología. La capa externa es compacta en casi todos los taxones, muy fina en $B$. corralense, $B$. mochaenum subsp. achalense, $B$. microphyllum y $B$. penna-marina, con mayor espesor en $B$. mochaenum subsp. mochaenum y $B$. blechnoides. La capa media es compacta en $B$. corralense, B. mochaenum subs. achalense, $B$. spicant y B. stoloniferum, laminar o "foliosa" en $B$. microphyllum y B. penna-marina y fibroso-fenestrada en $B$. mochaenum subsp. mochaenum y B. blechnoides.

La micro-ornamentación está presente en algunos taxones. Es microgranular fina ( $B$. corralense) o gruesa (B. mochaenum subsp. squamipes), en forma de microgránulos homogéneos, dispuestos densamente o de forma esparcida e irregular (B. stoloniferum).

A continuación se describen pormenorizadamente los taxones del grupo. Los caracteres morfológicos más destacables y datos ecológicos de interés se reúnen en la tabla 1 .

\section{Blechnum asperum}

Observación con MO. Esporas monoletes, elipsoidales, de (40) 53 (65) x (32) 41 (48) $\mu \mathrm{m}$, relación diámetro mayor / diámetro menor 1,29. Esporodermo de 4-5 $\mu \mathrm{m}$ de espesor. Perisporio castaño, rugado a rugado-crestado, con crestas bajas, con apariencia quebradiza sobre la superficie del exosporio. Exosporio amarillento. Lesura fina, de (27) 32 (40)' $\mu \mathrm{m}$.

Ejemplares de referencia. CHILE: Concepción: Arauco, Isla Mocha, WeldtRodríguez 1085/378- B (CONC).

\section{Blechnum blechnoides (fig. 2 E-F)}

Observación con MO. Esporas monoletes, castaño-amarillentas, de contorno elipsoidal en vista polar, de (40) 49 (60) x (26) 40 (45) $\mu \mathrm{m}$, relación diámetro mayor / diámetro menor 1,22. Cara proximal algo convexa en vista ecuatorial. Esporodermo de 3-4 $\mu \mathrm{m}$ de espesor. Observación con MEB. Perisporio con ornamentación rugada a rugado-fosulada, con muros bajos definidos, como pliegues gruesos de disposición más bien ordenada. Esta ornamentación se distribuye regularmente en toda la superficie, incluso en la zona de la lesura, con muros poco elevados que se anastomosan y forman muros más alargados. Debajo de la superficie, que es compacta, se observa una capa con estructura fibrosofenestrada (fig. 2 F). La lesura tiene unas partes del largo mayor de la espora, mide (20) 23 (27) $\mu \mathrm{m}$ y sobresale moderadamente del contorno.

Ejemplares de referencia: CHILE: Concepción: Talcahuano, Rocoto, en acantilados, de la Sota 6024 (LP). Talcahuano, Caleta Tumbes, en gruta, Junge 5365 (2 ejemplares LP). Valdivia: Cordillera Pelada, Escuche 368 (LP). Cordillera Pelada, cerca Huicolla, 50 m, de la Sota 2773 (LP). Corral: 


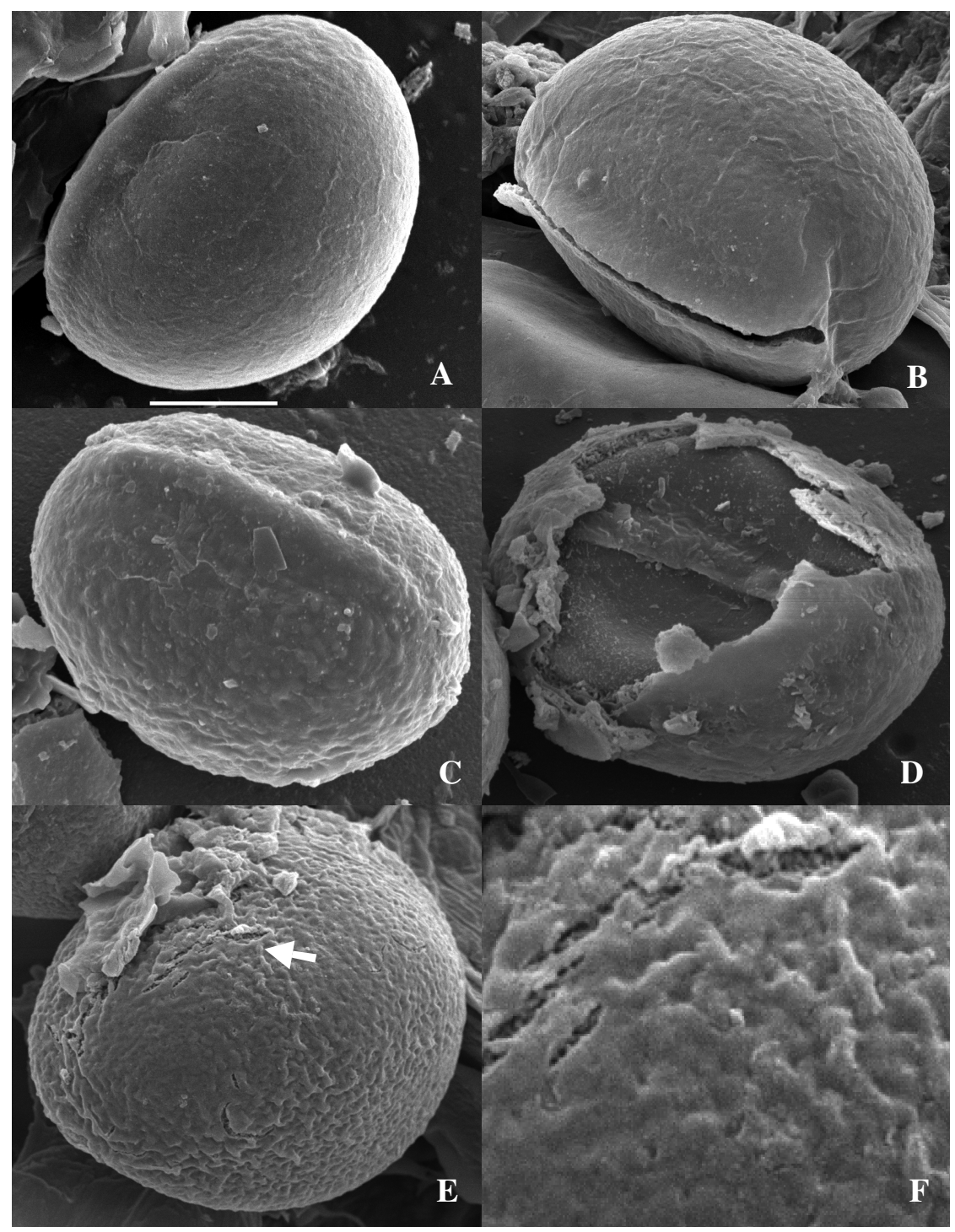

Figura 2. Caracteres esporales en el grupo Blechnum penna-marina: B. mochaenum subsp. mochaenum y B. blechnoides. A-C: B. mochaenum subsp. mochaenum. A: Vista lateral con lesura prominente curva, en material de Argentina, Neuquén, Gentili 814 (LP). B: Lesura abierta, en material de Argentina, Neuquén, Diem 1192 (LP). C: Perisporio rugulado, lesura curva en material de Argentina, Chubut, Roig et al. 13907 (LP). D: Perisporio y exosporio en espora fracturada, en material de Argentina, Neuquén, Gentili 814 (LP). E: B. blechnoides, de Chile, Llanquihue, Parra 11056 (LP). F: Detalle en E. Barra $=10 \mu \mathrm{m}$ en A-E; $5 \mu \mathrm{m}$ en F. Characters of the spores of the Blechnum penna-marina group: B. mochaenum subsp. mochaenum and B. blechnoides. A-C: B. mochaenum subsp. mochaenum. A: Spore in lateral view with curve, protrudent laesura, from Argentina, Neuquén, Gentili 814 (LP). B: Spore with open laesura, from Argentina, Neuquén, Diem 1192 (LP). C: Spore with rugulate perispore, and curve laesura, from Argentina, Chubut, Roig et al. 13907 (LP). D: Perispore and exospore in a naturally broken spore, from Argentina, Neuquén, Gentili 814 (LP). E: B. blechnoides, from Chile, Llanquihue, Parra 11056 (LP). F: Detail in E. Bar= 10 um en A-E; 5 um en F. 
La Aguada, en quebrada, Gunckel 2880 (LP). Corral: La Rama, Gunckel 2336 (LP). Mehuín, en playa, "crece en agua, sobre rocas, a la orilla de la playa", Godoy s. $n$. (LP). Llanquihue: Huinay, Parra 11056 (LP).

\section{Blechnum corralense (figs. 1 A-B)}

Observación con MO. Esporas monoletes, castaño amarillento, de contorno elipsoidal en vista polar, de (55) 57 (60) x (32) 36 (40) $\mu \mathrm{m}$, con una relación diámetro mayor / diámetro menor de 1,55. Cara proximal marcadamente convexa en VE, con extremos muy redondeados. Esporodermo de $4 \mu \mathrm{m}$ de espesor. Perisporio con ondulaciones suaves y microgránulos finos, con una capa fibroso-fenestrada por debajo.

Observación con MEB. Perisporio subliso a rugulado- fosulado compacto, con ondulaciones amplias, poco profundas, con micropliegues tenues, espaciados en toda la superficie y con algunas microperforaciones, cubierto por micrornamentación granular homogénea y densa (fig. 1 B). La microornamentación es menos densa en la cara distal y en la zona de la lesura, donde los microgránulos se disponen en forma más desordenada. La lesura es tenuimaginada, mide 53 (56) $60 \mu \mathrm{m}$ y ocupa casi todo el largo de la cara proximal de la espora.

Ejemplares de referencia- CHILE: Valdivia: Corral: Casa Socorros, Gunckel 4887 (LP). Corral San Juan, Gunckel 1961(LIL). Valdivia, "en grutas" sin loc., Gunkel 15615 (LIL).

\section{Blechnum fernandezianum (fig. 6 A-C)}

Observación con MO. Esporas monoletes, de color castaño amarillento, contorno elipsoidal en vista polar, de (40) 50 (60) x (34) 36 (38) $\mu \mathrm{m}$, con relación diámetro mayor / diámetro menor de 1,38. Cara proximal es levemente convexa en VE. Esporodermo de $3 \mu \mathrm{m}$ de espesor. La superficie del perisporio es psilada con gránulos discretos dispuestos irregularmente. Observación con $M E B$. Perisporio psilado con orbículas esferoidales dispuestas con densidad y disposiciones variables. El contorno de las orbículas es relativamente constante y su superficie rugosa a microgranular. La lesura mide (20) 23 (27) $\mu \mathrm{m}$ es corta, delgada, muy tenue y no se extiende por más de $1 / 3$ de la cara proximal de la espora.

Ejemplares de referencia: CHILE: Juan Fernández: Isla Robinson Crusoe (ex Más Afuera), Quebrada de la Mata Maqui, on trail to Correspondencia Camp, Meyer $9437 \mathrm{y}$ 9745 (LP). End of Casas Canyon, Solbrig et al. 3694 (LP). Isla Marinero Alejandro Selkirk (ex Masatierra), Pangal, abundant in Maqui forest, Solbrig et al. 3822 ( LP). Pangal Falls, Meyer 9594 (LP). Subida al Mirador de Selkirk, desde San Juan Bautista, Agua del Guindal, $260 \mathrm{~m}$, Marticorena et al. 9002 (MA).

\section{Blechnum microphyllum (fig. 4 E-F)}

Observación con MO. Esporas monoletes, amarillentas, de contorno elipsoidal, de (35) 48 (55) x (26) 30 (36) $\mu \mathrm{m}$, con una relación diámetro mayor / diámetro menor de 1,6. Cara proximal levemente convexa a recta en VE. Esporodermo de 55,2 $\mu \mathrm{m}$ de espesor. La ornamentación es rugulada a levemente plegada.

Observación con MEB. El perisporio tiene una superficie venulosa a venulosorugulada o venuloso-laminar, con pliegues irregulares muy delgados, densos o dispersos y algunos procesos como microprominencias muy dispersas (fig. $4 \mathrm{~F}$ ). La capa externa es comparativamente muy delgada, se desprende en laminillas (fig. 4 E) y por debajo se observa la capa media con estructura laminar a "foliosa", con laminillas densamente e irregularmente entrecruzadas, muy características, similares a las que se presentan en B. penna-marina (fig. 4 A-D). 


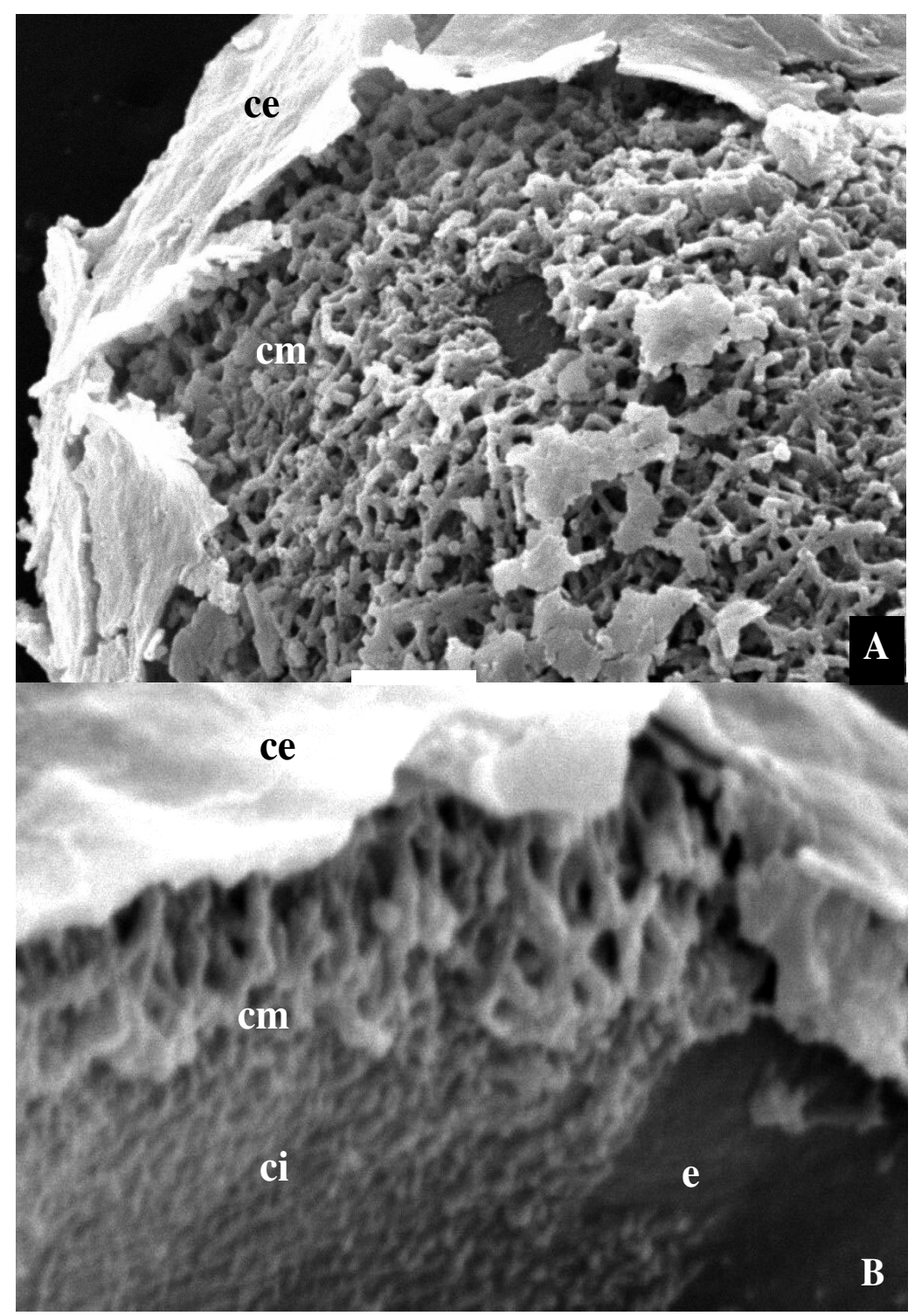

Figura 3. Caracteres esporales en el grupo Blechnum penna-marina: B. mochaenum subsp. mochaenum. A-B: Vistas del perisporio en cortes y del exosporio en superficie, en material de Argentina, Neuquén, Gentili 814 (LP). A: Capas del perisporio, capa externa rota, capa media fenestrado-alveolar. B: Capas del perisporio en corte, restos de la capa interna adherida al exosporio y exosporio. ce, capa externa; cm, capa media; ci, capa interna; e: exosporio. Barra: $2 \mu \mathrm{m}$. Characters of the spores of the Blechnum penna-marina group: B. mochaenum subsp. mochaenum. A-B: Transversal section of the perispore and surface of exospore, in material from Argentina, Neuquén, Gentili 814 (LP). A: Layers of the perispore, broken external layer in top, medium fenestrate-alevolate layer below. B: Section of the perispore with layers, remains of the internal layer over the exospore, and partial view of the smooth exopore. ce, external layer; cm, medium layer; ci, internal layer; e: exospore. Bar: 2 um. 
La lesura mide (32) 44 (50) es larga, alcanza más de los $2 / 3$ de la cara proximal y sobresale como un pliegue angosto y a veces algo filoso.

Ejemplares de referencia: ARGENTINA: Neuquén: Kurtz, F. 6249 (CORD). Río Agrio, Lago Caviahué, 1700 m, Diem 3366 (LP). Cavihué, Kuhnemann 24 (LP). Copahue, Cabrera et al. 22898 (LP) y Monticelli $s / n$ (LP). Aluminé: Ruta provincial 11, $23 \mathrm{~km} \mathrm{~N}$ del Lago Ñorquinco, hacia Paso Icalum, Correa et al. 10069 (LP). CHILE: Bío-Bío: Laguna de Las Lajas, Faldas de Sierra Velluda, Ricardi \& Marticorena 26218 (CONC, LP). Maule: 800-1500 m, 35.51 S 71.06 W, Arroyo, M. T. K. et al. 20114 (CONC; SGO). Nuble: Termas de Chillán, región del Bío-Bío, Pedersen 14240 (LP).

\section{Blechnum mochaenum subsp. mochaenum}

(figs. 2 A-D y 3 A-B)

Observación con MO. Esporas monoletes, castaño amarillento, de contorno elipsoidal en vista polar, de (40) 44 (48) x (26) 30 (32) $\mu \mathrm{m}$, con una relación diámetro mayor/diámetro menor de 1,33 en la mayoría de los ejemplares estudiados y de (50) 54 (58) x (32) 35 (46) $\mu \mathrm{m}$, con una relación diámetro mayor / diámetro menor de 1,42 en los ejemplares de Argentina, Neuquén, Diem 1192 (LP) y Chubut, Roig et al. 13907 (LP). Esporodermo de 5-7 $\mu \mathrm{m}$ de espesor. Cara proximal es convexa en VE, con ornamentación microgranular.

Observación con MEB. El perisporio es rugado, rugulado o rugado-fosulado (fig. $2 \mathrm{~A}-$ C), con la ornamentación uniformemente distribuida y sin micro-ornamentación. La capa externa es compacta y la capa media fibrosofenestrada a fibroso-alveolar (fig. 3 A-B). La capa interna es poco visible y aparentemente tiene estructura granular (fig. 3 B). El exosporio es liso a microgranular (fig. 3 B). La lesura mide (30) 34 (43) $\mu \mathrm{m}$, es casi larga o tan larga como la cara proximal y sobresale del contorno.

Ejemplares de referencia: ARGENTINA:
Neuquén: margen oriental del Lago Queñi, 820 m, Gentili 814 (LP). Isla Victoria, Diem 1192 (LP). Cascada de Chochín, senda a Chochín, desvío del camino a Hua-hum, Prada s/n (LP, MA). Camino a Paimún, Prada $s / n$ (LP, MA). Lago Nahuel Huapi, Brazo Blest, Puerto Blest, camino a la cascada de los Cántaros, Prada s/ $n$ (LP, MA). Chubut: Futaleufú: Parque Nacional Los Alerces, Lago Cisnes, Roig et al. 13907 (LP). Río Negro: Laguna Frías, Conte 16 (LP). 'CHILE: Arauco: Isla de Mocha: camino entre el Faro Oeste y La Hacienda, Cordón Central, Weldt-Rodríguez 1111/406 (CONC 34368, LP).Concepción: Coronel Santa Juana: Duralí, Rodríguez Ríos 2 (CONC 33807, LP). Talcahuano: Caleta Tumbes, de la Sota 6015 (LP). Llanquihue: Lago Puelo, Parra 11282 (LP). PEULLA, Pfister 13554 (LP). Malleco: Parque Nacional Contulmo, de la Sota 6009 (LP). Osorno: Parque Nacional Puyehué, Roig 13662 (LP) y Roig et al. 13659 (LP). Aguas Calientes, Debandi 9 (LP). IX REGIÓN: Cunco, Lago Colicó, Debandi 51 (LP).

\section{Blechnum mochaenum subsp. achalense} (fig. 1 C-D)

Observación con MO. Esporas monoletes, amarillentas, de contorno ellipsoidal en vista polar, de (52) 50 (55) x (30) 33 (35) $\mu \mathrm{m}$, con una 'relación diámetro mayor / diámetro menor de 1,66. Esporodermo de 3-4 um de espesor. Cara proximal plana en VE. La ornamentación es sublisa, microgranular.

Observación con MEB. El perisporio es algo ondulado a rugulado, con algunas laminillas dispersas y con abundantes microgránulos homogéneos, densamente dispuestos (fig. $1 \mathrm{D}$ ). El perisporio tiene una capa externa muy delgada y una interna compacta, aproximadamente 2-3 veces más gruesa que la externa. El exosporio es casi liso, con microgránulos abundantes y homogéneamente distribuidos sobre una superficie subondulada. La lesura, de la 


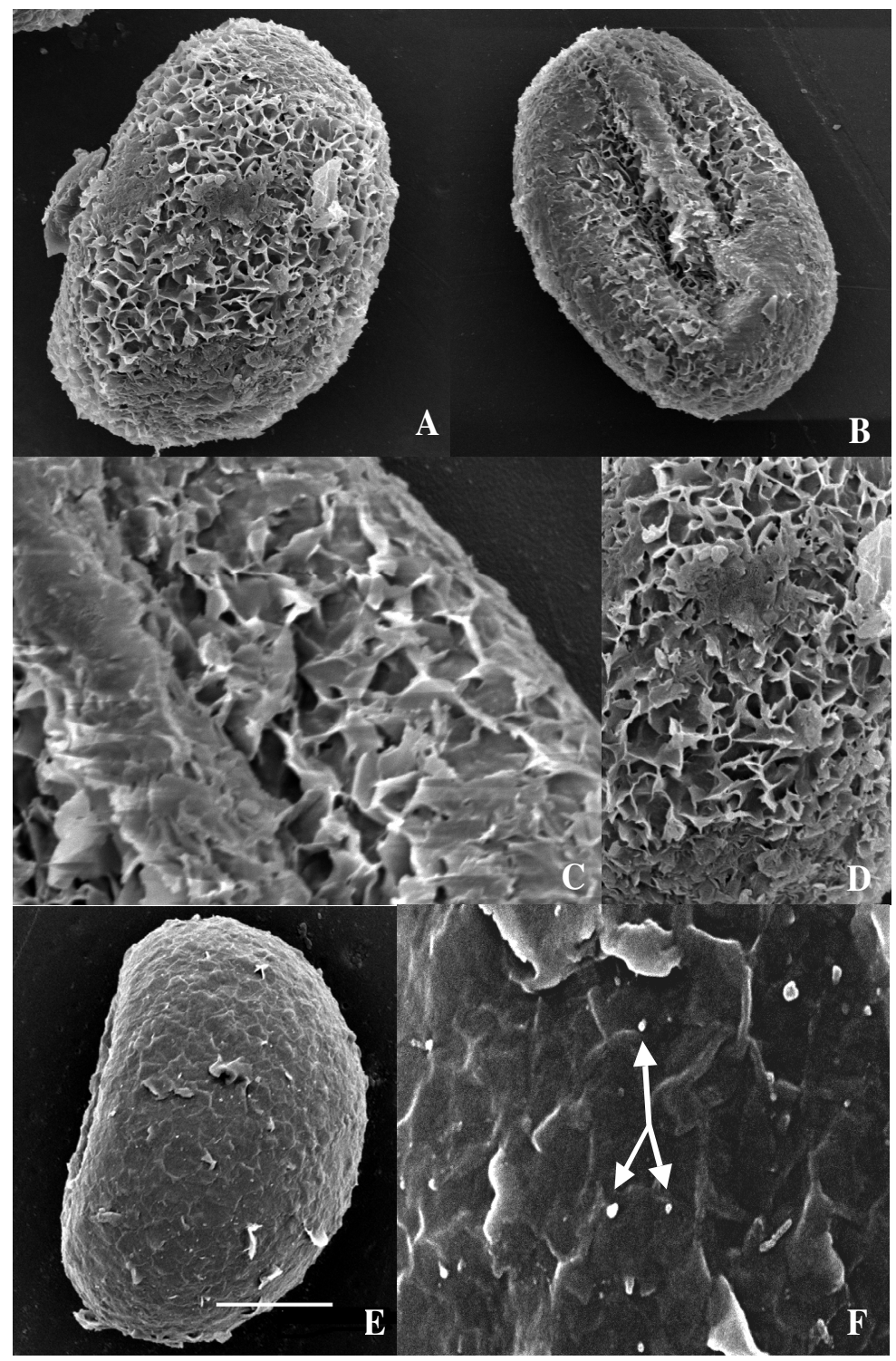

Figura 4. Caracteres esporales en el grupo Blechnum penna-marina: B. penna-marina y B. microphyllum. A-D: B. penna-marina, de Argentina, Tierra del Fuego, Torres 1122 (LP). A: Vista ecuatorial, perisporio con capa externa delgada rota y capa media folioso lamelar visible. B: Lesura. C-D: Detalles de la estructura de la capa media folioso lamelar. E-F: B. microphyllum, de Argentina, Neuquén, Diem 3366 (LP). E: Vista lateral con perisporio. F: Detalle del perisporio en E. Barra $=10$ $\mu \mathrm{m}$ en A-B, E; $2 \mu \mathrm{m}$ en C-D, F. Characters of the spores of the Blechnum penna-marina group: B. penna-marina and B. microphyllum. A-D:B. penna-marina, from Argentina, Tierra del Fuego, Torres 1122 (LP). A: An equetorial view of a spore with the thin, broken external layer, and the medium, "foliose", lamelar layer below. B: Laesura. C-D: Details of the structure of the medium, folioselamelar layer. E-F: B. microphyllum, from Argentina, Neuquén, Diem 3366 (LP). E: Spore with perispore, lateral view. $F$ : Detail of the perispore in $E$. Bar $=10 \mu \mathrm{m}$ en $A-B, E ; 2 \mu m$ en $C-D, F$. 
misma longitud de la cara proximal, (52) 49 (55) sobresale del contorno de la espora (fig. $1 \mathrm{C})$.

Ejemplares de referencia: ARGENTINA: Chubut: Lago $\mathrm{N}^{\circ}$ 1, Kühenmann 53 (BA). Parque Nacional Los Alerces, Lago Cisne, Pérez Moreau s/n (BA 49470). Neuquén: Parque Nacional Nahuel Huapi, Ventisqueros, Núñez s/n (BA 56522). Los Lagos, Nahuel Huapi, Quetrihué, Lago Patagua, de la Sota 2164 (BA). Salta: Santa Victoria, Los Toldos, Finca El Nogalar, Río Huaico, 1800 m, Cassá et al. 235 (LP) -planta de la izquierda-.

\section{Blechnum mochaenum subsp. squamipes} (fig. 5 A-D)

Observación con MO. Esporas monoletes, amarillentas, de contorno elipsoidal en vista polar, de (37) 45 (48) x (27) 32 (40)' $\mu \mathrm{m}$, con una relación diámetro mayor / diámetro menor de 1,37. Esporodermo de 5-5,1 $\mu \mathrm{m}$ de espesor. Cara proximal recta en VE. La ornamentación es subcrestada a crestado-reticulada.

Observación con MEB. El perisporio presenta muros, pliegues o crestas bajos que delimitan aréolas más o menos subpoligonales cubiertas por micrornamentación granular homogénea densa (fig. $5 \mathrm{~A}, \mathrm{C}$ ). Las crestas o pliegues bajos se ordenan en forma de retículo incompleto, delimitando áreas cubiertas densamente por microgránulos (fig. 5 C). En esporas juveniles estas áreas están menos definidas, son más amplias, los pliegues son menos próximos y no se presenta la micro-ornamentación (Fig. 5 D). En esporas jóvenes la capa externa es compacta y por debajo se observa una estructura fibroso-fenestrada similar a la de B. mochaenum subsp. mochaenum aunque más delgada. La lesura mide (28) 32 (36) $\mu \mathrm{m}$. Ejemplares de referencia: ARGENTINA: Catamarca: Andalgalá, Esquina Grande, Joergensen 1571 (LP, LIL, US). Córdoba: Sierra Grande: Cuesta del Mogote, Castellanos s. n. (LIL 37924).
Jujuy: El Cucho, Cerro labrado, Potrero de las Mulas, de la Sota 4373 (LP, US). Salta: Santa Victoria, Los Toldos, Finca El Nogalar, Río Huaico, $1800 \mathrm{~m}$ (saxícola, en área aterrazada, sobre musgos), Cassá et. al. 255 (LP)-planta de la derecha-. Tucumán: Quebrada Pueblo Viejo, de la Sota 4070 (LP). Estancia Las Pavas, Puesto E1 Saladillo, Venturi 3001 (LIL).

\section{Blechnum penna-marina (fig. 4 A-D)}

Observación con MO. Esporas monoletes, amarillentas, de contorno elipsoidal en vista polar, de (37) 44 (48) x (26) 31 (38) $\mu \mathrm{m}$, con una relación diámetro mayor / diámetro menor de 1,4 en la mayoría de los ejemplares estudiados, y de (45) 48 (52) x (30) 36 (38) $\mu \mathrm{m}$, con una relación diámetro mayor / diámetro menor de 1,3 en los ejemplares de Argentina, Neuquén, Gómez Rozas 503 (LP) y Tierra del Fuego, Torres 1122 (LP). Espordermo de 4-5 $\mu \mathrm{m}$ de espesor. Cara proximal casi recta en VE. La ornamentación es irregularmente venulosorugulada.

Observación con MEB. El perisporio tiene posee una capa externa irregularmente venuloso rugulada, muy fina, fácilmente caduca, que se separa con facilidad en laminillas muy delgadas permitiendo observar la capa media (fig. 4 A-B). La capa media tiene una estructura fenestradolaminar o fenestrado alveolar, con laminillas entrecruzadas muy características (fig. $4 \mathrm{C}$ D); se adhiere fuertemente al exosporio, que presenta ornamentación microgranular. La lesura mide (25) 30 (34) $\mu \mathrm{m}$, no sobresale del contorno de la espora y ocupa la æ partes de la longitud de la cara proximal.

$$
\text { Ejemplares de referencia: }
$$

ARGENTINA: Chubut: Krapovickas, A. 4029 (LP). Chubut: Koslowsky, J., s.n. (LP19116). Córdoba: Minas: Sierras Grandes, Gautier s/n. (LP). Jujuy: de la Sota, E. R. 2895 (LP). Neuquén: Huiliches: Baños de 


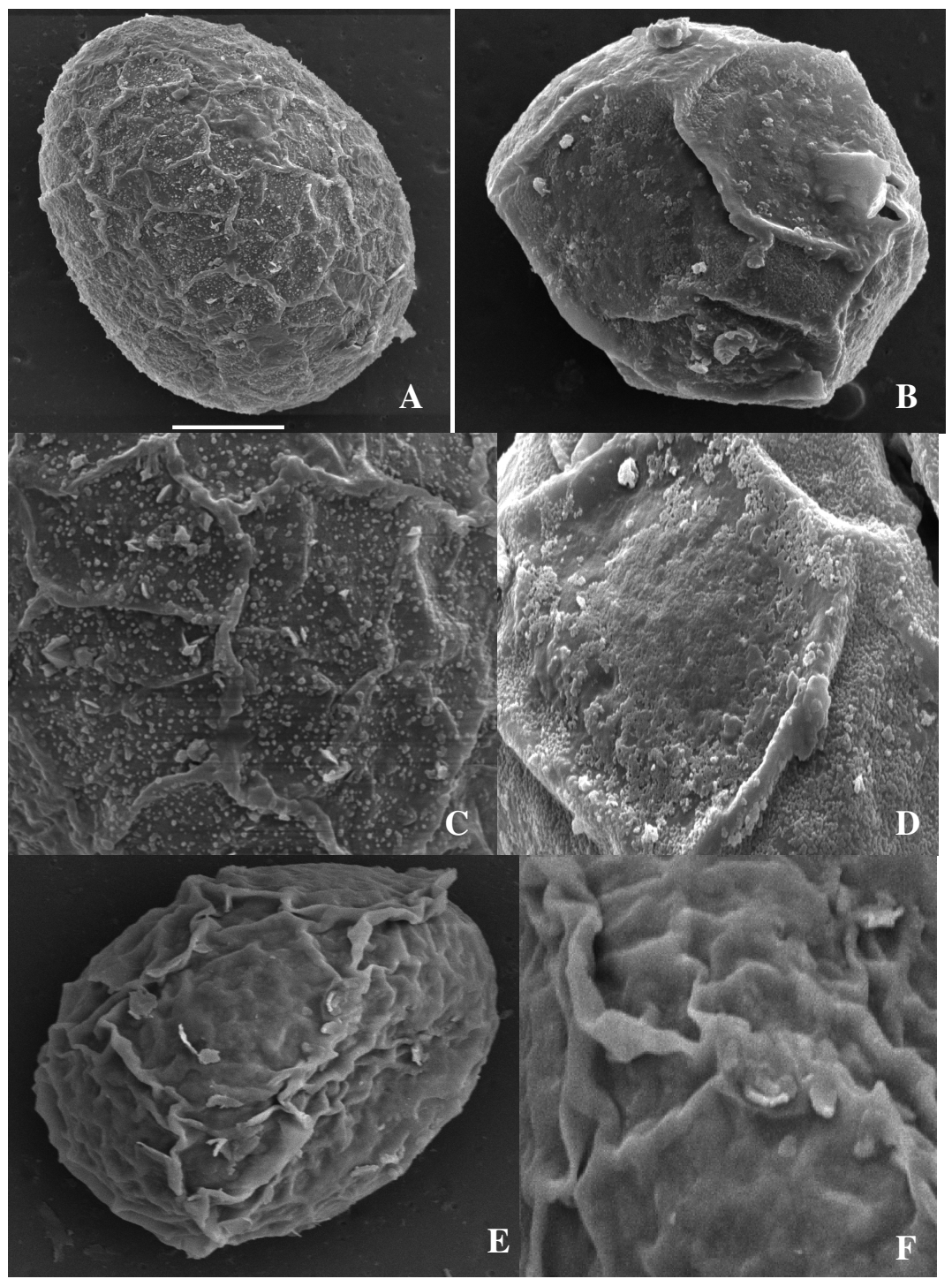

Figura 5. Caracteres esporales en el grupo Blechnum penna-marina: B. mochaenum subsp. squamipes y B. spicant. A-D: B. mochaenum subsp. squamipes de Córdoba, Castellanos 488 (BA). A: Espora adulta. B: Espora juvenil de una tétrada. C: Detalle de la ornamentación en A, perisporio con microgránulos y pliegues subreticulados. D: Detalle de la ornamentación en B, perisporio inmaduro. E-F: B. spicant, de España, Galicia, Prada $s / n$ (MACB). E: Espora con perisporio con pliegues irregulares. F: Detalle de pliegues. Barra $=10 \mu \mathrm{m}$ en A, E; $15 \mu \mathrm{m}$ en B; $5 \mu \mathrm{m}$ en D, F. Characters of the spores of the Blechnum penna-marina group: B. mochaenum subsp. squamipes and B. spicant. AD: B. mochaenum subsp. squamipes from Córdoba, Castellanos 488 (BA). A: Adult spore. B: Juvenile spore from a tetrad. C: Detail of macro-ornamentation in A, perispore folded to reticulate-cristate, with abundant, coarse micro-granules. D: Detail of macro-ornamentation in B, spore with inmature perispore. E-F: B. spicant, from España, Galicia, Prada s/n (MACB). E: Spore with folded perispore, irregularly distributed folds. F: Detail of folds in E. Bar= $10 \mu \mathrm{m}$ en $A, E ; 15 \mu \mathrm{m}$ en $B ; 5$ um en D, F. 
Lahuén-Có, Gómez \& Rosas 503 (LP). Río Negro: Río Manso, al pie del Tronador, cascada Nacimiento, 110 m, Diem 3087 (LP). Tierra del Fuego: Puerto Cook, Torres 1122 (LP). Estancia Moat, 19-03-05, ruta provincial J, $105 \mathrm{M}$, Rodríguez et al. s. $n$. (LP, MA). Estancia Marina, 12-03-05, Prada et al. s. n. (LP, MA). BOLIVIA: La Paz: Larecaja, salida de Sorata en dirección a Consata, $3450 \mathrm{~m}$, Fernández Casas \& Molero 6582 (MA). BRASIL: Santa Catarina: Lages, Spannagel s. n. (LIL 474029). CHILE: X Región: Provincia Palena, 10,4 km SE of Puerto Ramírez on road to Palena, Stuessey et al. 7181 (MA). XII Región: Provincia Tierra del Fuego, Sector Río Cóndor, Forestal Trillium, Pisano et al. 7910 (MA).

\section{Blechnum spicant (figs. 5 E-F)}

Observación con MO. Esporas monoletes, amarillentas, de contorno esferoidal en

vista polar, de (46) 50 (52) x (32) 35

(37) $\mu \mathrm{m}$, con una relación diámetro mayor / diámetro menor de 1,43. Esporodermo de 3 $4 \mu \mathrm{m}$ de espesor. Cara proximal recta en VE. La ornamentación es sublisa a plegada.

Observación con $M E B$. El perisporio es compacto, casi liso o plegado. Los pliegues no delimitan aréolas, son gruesos o finos, irregulares, redondeados o afilados y planos (fig. 5 E-F). Su densidad varía en esporas de un mismo ejemplar y de un ejemplar a otro. El exosporio es psilado y acompaña al perisporio en la elevación a nivel de la lesura. La lesura, de (45) 48 (51) $\mu \mathrm{m}$ es larga y alcanza unas æ partes de la longitud de la cara proximal de la espora; es algo afilada y sobresale levemente del contorno. No se observó micrornamentación.

Ejemplares de referencia: DINAMARCA: Aarhus: Faurholt heath near Frederiksharn, Jensen, Nielsen \& Pedersen 461 (BA 66805). ESPAÑA: Galicia: Coruña,
Fraga de Caaveiro, Prada $s / n$ (MACB). Orense, Valle de Valdeorras, El Mazo, Prada $s / n$ (MACB). SUIZA: Flore du pays d'Enhaut: Les Mosses, bois clear, Pedersen 2486 (LP). ESTADOS UNIDOS DE NORTEAMERICA: Washington State, Seatle, s. coll. (BA 13838).

\section{Blechnum stoloniferum (fig. 1 E-F)}

Observación con MO. Esporas monoletes, amarillentas, de contorno esferoidal en vista polar, de (45) 51 (54) x (30) 36 (46) $\mu \mathrm{m}$, con una relación diámetro mayor / diámetro menor de 1,42. Esporodermo de $4 \mu \mathrm{m}$ de espesor. Cara proximal ligeramente convexa en VE. La ornamentación es psilada.

Observación con $M E B$. El perisporio es rugado con microgránulos y algunos pliegues poco elevados. La capa interna del perisporio es compacta. El exosporio presenta ornamentación rugada microgranular. La lesura mide (34) 37 (40) $\mu \mathrm{m}$ y alcanza una longitud de $3 / 4$ partes de la cara proximal de la espora.

Ejemplares de referencia: MÉXICO: Oaxaca: Mixes, NW slopes of Cerro Zempaltepetl, trail from Yacoche to Tototepec, Mickel \& Leonard 4661 (LP, NY). Teotitlán, 26-29 km NE of Teotitlán del Camino, vicinity of Pass at Puerto Soledad, Mickel \& Hellwig 4104 (LP, NY). Hidalgo: Pringle 13808 (MO).

\section{DISCUSIÓN}

Las esporas de los taxones reunidos en el grupo B. penna-marina (Rolleri \& Prada, 2006 b) presentan variaciones morfológicas en el esporodermo que permiten distinguirlas a nivel específico e infraspecífico.

La pared esporal está formada por exosporio y perisporio. El perisporio siempre se encuentra presente y es la capa más 


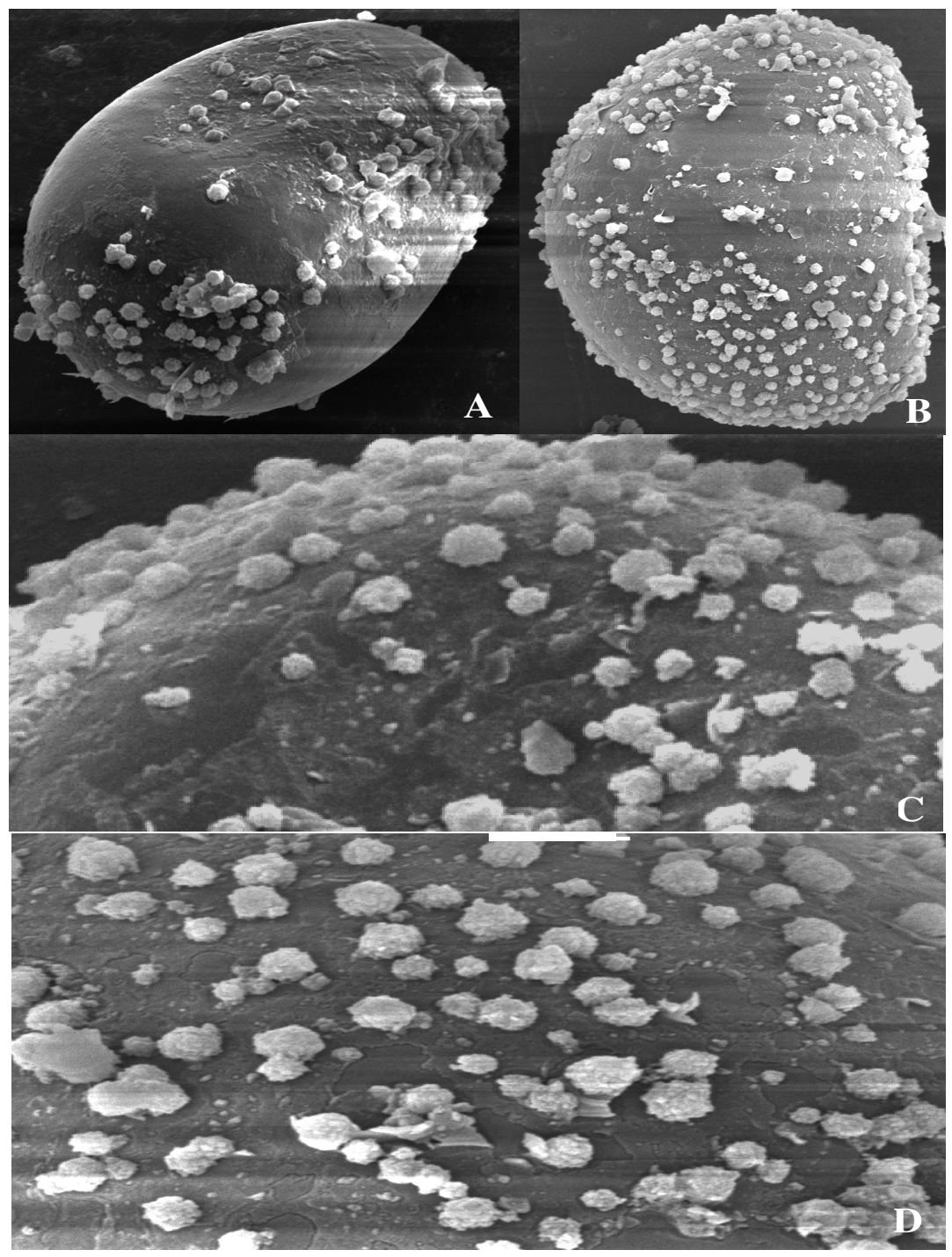

Figura 6. Caracteres esporales en el grupo Blechnum penna-marina: B. fernandezianum y comparación con B. serrulatum. A, C: B. fernandezianum de Archipiélago de Juan Fernández, Isla Robinson Crusoe, Meyer 9745 (LP). A: Espora completa, con perisporio psilado, orbículas dispersas y lesura corta, muy tenue. C: Detalle de orbículas en A. B, D: B. serrulatum, de Argentina, Chaco, Schulz 9 (SI). B: Espora completa en vista lateral, con perisporio levemente rugado, orbículas irregularmente esparcidas y lesura poco sobresaliente. D: Detalle de orbículas en B. Barra= $6 \mu \mathrm{m}$ en A; 7,5 $\mu \mathrm{m}$ en B; 1,7 $\mu \mathrm{m}$ en $\mathrm{C} ; 2 \mu \mathrm{m}$ en D. Characters of the spores of the Blechnum penna-marina group: B. fernandezianum and a comparison with B. serrulatum spores. A, C: B. fernandezianum from the Juan Fernández islands, Robinson Crusoe Island, Meyer 9745 (LP). A: Spore with psilate perispore bearing orbicules and a short, thin laesure. C: Detail of orbicules in A. B, D: B. serrulatum, from Argentina, Chaco, Schulz 9 (SI). B: Spore in lateral view with softly rugate perispore, irregularly distributed orbicules and thin laesura. D: Detail of the orbicules in B. Bar= $6 \mu \mathrm{m}$ en A; 7,5 $D$. 
desarrollada y la que aporta caracteres diagnósticos más importantes, ya que varía tanto en la superficie externa como en la estratificación. La macro-ornamentación es muriforme y hay perisporios lisos, rugulados, rugados, venuloso-rugulados, venulosolaminares o irregularmente plegados o crestado-reticulados. La única excepción es B. fernandezianum, con esporas psiladas cubiertas por orbículas esféricas rugosas. La micro-ornamentación se encontró en tres taxones, B. corralense, B. mochaenum subsp. achalense y $B$. stoloniferum, y es de tipo granular, fina o gruesa, más bien homogénea, densa o dispersa.

Desde el punto de vista de la estratificación, los perisporios son compactos en $B$. spicant y estratificados, con dos o tres capas, en los taxones restantes. De estas tres capas, dos se detectan fácilmente (la externa y la media), mientras que la interna no siempre se observa claramente. La capa externa es compacta en todos los taxones. La capa media es compacta en $B$. corralense, B. mochaenum subsp. achalense y $B$. stoloniferum o bien presenta ornamentación más o menos elaborada y es variadamente fibroso-fenestrada o fibroso-alveolar, como en B. blechnoides, $B$. mochaenum subesp. mochaenum y $B$. mochaenum subsp. squamipes, fenestradolaminar, con aspecto "folioso" en $B$. microphyllum y B. penna-marina. La interna, pegada al perisporio, aparece como finamente granular y es poco detectable. No hay relación entre el espesor y ornamentación del perisporio y la mayor o menor elaboración de las capas por debajo. Las combinaciones se producen al azar y parecen variar en los niveles específicos o infraspecíficos.

La capa media con estructura laminar o "foliosa" fue considerada como el rasgo más común en Blechnum (Tryon \& Lugardon, 1991), pero en el grupo sólo se encontró en B. microphyllum y B. penna marina. Fuera del grupo estudiado, Lloyd (1976) la describió para dos especies de Sadleria Kaulf., S. rigida Copel. y S. souleytiana (Gaud.) Moore y para Woodwardia areolata (L.) Moore. Este autor describió este tipo de perisporio como complejo, con estructura multiestratificada y lo interpretó como un rasgo primitivo. También se la observó en esporas de B. tabulare (Rolleri, Prada \& Passarelli, datos no publicados).

La capa media con estructura fibrosofenestrada, del tipo encontrado en las esporas de B. mochaenum subsp. mochaenum se ha podido observar en perisporios de $B$. cordatum (Desv.) Hieron., B. columbiense Hieron. y $B$. loxense (Kunth) Hook. ex Salomon, mientras que una capa media con pilares menos complejamente entrecruzados se puede hallar en esporas de $B$. brasiliense Desv. y de $B$. magellanicum (Desv.) Mett. (Rolleri, Prada \& Passarelli, datos no publicados).

Las esporas de $B$. fernandezianum tienen un tipo de ornamentación poco frecuente en Blechnum, con orbículas muy características. Tryon \& Tryon (1991) diferencian los llamados "glóbulos", producidos por el tapete al mismo tiempo que el exosporio, de las "esférulas" (orbículas) que se forman junto con el perisporio. En este sentido, las orbículas de $B$. fernandezianum corresponderían a la segunda categoría. Durán (1997) las llamó “depósitos esféricos superficiales" y también las mencionó para $B$. serrulatum Rich. pero no para $B$. fernandezianum. Las orbículas de $B$. fernandezianum son más grandes que las de $B$. serrulatum y tienen la superficie granular. No son exclusivas de'Blechnum y están presentes también en otros géneros de Polypodiaceae s. 1., como Platycerium Desv., Drynaria (Bory) Sm., Christiopteris Copel. y Polypodium L. (Tryon \& Lugardon, 1991). En relación con este aspecto, Lellinger \& Taylor (1997) llamaron la atención sobre el hecho de que los tipos de ornamentación de las esporas de pteridófitos pueden ser recurrentes y que taxones no vinculados 
entre sí ni afines pueden presentar una morfología similar del esporodermo, atribuyendo el fenómeno a convergencia o paralelismo en la evolución de las paredes esporales en el grupo.

Las esporas plegadas también son frecuentes en Blechnum y no están presentes solamente en B. spicant; este tipo de perisporio se ha registrado también en esporas de $B$. tabulare (Thunb.) Kuhn, B. magellanicum y otras especies similares de hábito arborescente (Rolleri, Prada \& Passarelli, datos no publicados). La presencia, tipos y distribución de los pliegues son variables y los perisporios externamente plegados se combinan con capas medias de estructura diferente, lo que sugiere, nuevamente, que los rasgos esporales se relacionan con las especies y no con los grupos establecidos hasta ahora.

Las esporas con muros o crestas bajas delimitando áreas más o menos regulares, que en el grupo caracterizan a $B$. mochaenum subsp. squamipes, también se presentan en otras especies neotropicales de Blechnum, como B. kunthianum C. Chr. y B. schottii (Colla) C. Chr. Sin embargo, los muros bajos de B. mochaenum subsp. squamipes delimitan aréolas más bien pequeñas y desiguales, cubiertas uniformemente con micrornamentación granular gruesa y homogénea, ausente o más dispersa en las especies neotropicales citadas (Passarelli \& Rolleri, datos no publicados).

Rolleri \& Prada (2006 b) utilizaron los caracteres esporales para cambiar el status de los taxones relacionados con B. mochaenum, considerando dos variedades como subespecies (B. mochaenum subsp. achalense y $B$. mochaenum subsp. squamipes) y elevando otra a la categoría de especie (B. fernandezianum), un criterio que este estudio tiende a confirmar como el adecuado. Las diferencias en la ornamentación y en la estructura del perisporio de las esporas de las subespecies de $B$. mochaenum son destacables y existen más diferencias entre las esporas de estos taxones que entre otros del grupo o de otros grupos.

Tryon \& Lugardon (1991) consideraron que las Blechnaceae se caracterizan por un perisporio elaborado que constituye la mayor parte del esporodermo. El grupo estudiado no puede definirse en conjunto por un tipo esporal, ni en lo relativo a la ornamentación externa del perisporio ni en lo que respecta a su estratificación. De lo aquí observado se deduce, más bien, que en el género Blechnum existen distintos grados de desarrollo del perisporio. Estas variaciones deberían correlacionarse con otros caracteres morfológicos, no esporales, para una mejor sistematización y para interpretar más adecuadamente las relaciones de las especies en el género, así como la historia evolutiva del grupo.

En general, los tamaños esporales son constantes en los taxones analizados. En dos ejemplares de B. mochaenum subsp. mochaenum y dos de B. penna-marina de Argentina se encontraron esporas de tamaño mayor que el observado en el resto de los especímenes estudiados. Esto podría relacionarse con diferencias de ploidía, ya que se trata de los mismos ejemplares en los que Rolleri \& Prada (2006 b) encontraron mayores tamaños de células epidérmicas y estomas. Los recuentos citológicos son escasos en el género y para los casos mencionados, los más recientes sugieren que ambos taxones son diploides con un número básico $\mathrm{x}=33$ (Jara-Seguel et al., 2006), aunque Manton \& Vida (1968) registraron un número $\mathrm{x}=34$ para material de $B$. pennamarina de Nueva Zelanda.

Finalmente, si se tienen en cuenta la diversidad de ambientes en los que crecen las especies estudiadas de Blechnum, no puede decirse por el momento que haya una relación entre los caracteres esporales y la ecología de las plantas. 
AGRADECIMIENTOS. Este trabajo fue realizado en el Laboratorio de Anatomía Vegetal Evolutiva y Sistemática (LEAVES), de la Facultad de Ciencias Naturales y Museo de La Plata, con el apoyo del Programa de Incentivos para Docentes Investigadores de la Universidad Nacional de La Plata (Argentina) y de la Agencia Española de Cooperación Internacional para la realización de un proyecto conjunto entre España y la Argentina (Resolucion 21-12-2006- A/6307/ 06) que brindó el apoyo para efectuar los viajes de campo y la recolección de material. La autora desea agradecer al Dr. Raúl Perdomo, Vicerrector de la Universidad de La Plata, por el apoyo brindado en el curso del desarrollo del proyecto y para los estudios de campo y especialmente, a la Dra. Cristina H. Rolleri ( FCNyM, Argentina) y Dra. Carmen Prada (UCM, Madrid, España) por la lectura crítica y las valiosas sugerencias hechas al manuscrito.

\section{BIBLIOGRAFÍA}

DURÁN, M. L. -1997- Estudios morfólogicos, taxonómicos y biosistemáticos en el género Blechnum. Blechnaceae-Pteridophyta. Tesis Doctoral, inéd. Facultad de Ciencias Exactas, Físicas y Naturales. Universidad Nacional de Córdoba. Argentina.

HOLMGREN, P.K.,N. H. HOLMGREN \& L.C. BARNETT -1990- Index Herbariorum, Part. I: The Herbaria of the World, 8th ed. New York Botanical Garden.

JARA-SEGUEL, P., M. ROMERO-MIERES y C. PALMA-ROJAS -2006- Números cromosómicos de Pteridófitos chilenos: primera contribución. Gayana Botánica 63(1): 115-118.

LELLINGER, D. B. -2002- A Modern Multilingual Glossary of taxonomic Pteridology. Pteridologia 3, 246 pp.

LELLINGER, D. B. \& W.C. TAYLOR- 1997- $A$ classification of spore ornamentation in the Pteridophyta, pp. 33-42, en R. J. Johns (ed.), Holttum Memorial Volume. Royal Botanic Gardens, Kew.

LLOYD, R. M. -1976- Spore morphology of the Hawaiian genus Sadleria (Blechnaceae). Amer. Fern J. 66: 1-7

LUGARDON, B. -1965- Structure des parois de la spore de Blechnum spicant (L.) Roth.Pollen et spores 7 (3): 409-428.
LUGARDON, B. -1974- La structure fine del'exospore et de la périspore des Filicinées'isosporées, II. Filicales. Commentaires.Pollen et Spores 16 (2): 161 226.

MANTON, I. J. \& J. D.VIDA -1968- Cytology of the fern flora of Tristan da Cunha. Proceedings of the Royal Society of London, Botany 170: 361-379.

MORBELLI, M. A -1976- Estudio palinológico de las especies austrosudamericanas del género Blechnum L., subgénero Blechnum (Blechnaceae-Pteridophyta). Bol. Soc.Argent. Bot. 17: 5-24.

PONCE, M. M. -1996- Pteridophyta. En: Zuloaga FO \& O Morrone (eds), Catálogo de las plantas vasculares de la República Argentina, I. Monographs in Systematic Botany, Missouri Botanical Garden 60: 1-79.

RAMOS GIACOSA, J. P, G. E. GIÚDICE, M. A. MORBELLI y E. R. SOTA de la - 2006aEstudio palonológico en Blechnum sect. Lomariocycas de Sudamérica austral. Revista del Museo Argentino de Ciencias Naturales, Resúmenes del XIII Simposio Argentino de Paleobotánica y Palinología: 114.

RAMOS GIACOSA, J. P, G. E. GIÚDICE \& M. A. MORBELLI.- 2006 b- Estudio palinológico de las Blechnaceae (Pteridophyta) el noroeste de Argentina. Revista del Museo Argentino de Ciencias Naturales, Resúmenes del XIII Simposio Argentino de Paleobotánica y Palinología: 115.

ROLLERI, C. \& C. PRADA.- 2006 a- Catálogo comentado de las especies mesoamericanas y sudamericanas de Blechnum L.,BlechnaceaePteridophyta. Anal. J. Bot. Madrid 63 (1): 67106.

ROLLERI, C. y C. PRADA -2006 b- Revisión de los grupos de especies del género Blechnum (Blechnaceae-Pteridophyta): el grupo B. pennamarina. Acta Bot.Malacitana 31: 7-50.

TRYON, A. F. \& L. LUGARDON.- 1991- Spores of the Pteridophytes: surface, wall structure, and diversity based on electron microscope studies. Springer-Verlag, Nueva York.

TRYON, R. M. \& A. TRYON -1982- Ferns and fern-allies from Tropical America. SpringerVerlag, Nueva York. 